\title{
Tow Minutes to Exclude or Diagnose SBP Using Leucocyte Esterase Reagent Strip
}

\author{
Authors \\ Ahmed A. Heikal ${ }^{1}$, Mahmoud M. El-Nokeety ${ }^{1}$, Amel E. Salem ${ }^{1}$, Eman Rushdy ${ }^{1}$, \\ Abeer Mohey ${ }^{2}$, \\ ${ }^{1}$ Internal Medicine Department, Cairo University Medical School, Cairo, Egypt \\ ${ }^{2}$ Clinical and Chemical Pathology, Cairo University Medical School, Cairo, Egypt ${ }^{2}$ \\ Corresponding Author \\ Dr Ahmed A.Heikal \\ Ass. Professor in Internal Medicine Department, Cairo University Medical School \\ Email:amin0880@yahoo.com, Ph:0201001960154
}

\begin{abstract}
Background: spontaneous bacterial peritonitis is the most frequent complication in HCV related liver cirrhosis causing significant mortality especially if there is a delay in diagnosis; the gold standard to diagnose spontaneous bacterial peritonitis (SBP) is a polymorphonuclear neutrophil count $\geq 250$ cells $/ \mathrm{mm}^{3}$ in ascitic fluid. This test is laborious and expensive. Urine reagent strips measuring leukocyte esterase activity have been proposed as a rapid and inexpensive alternative.

Aims: Is to emphasize the diagnostic accuracy of the Combi Stik leucocyte esterase reagent strips for rapid diagnosing SBP and to calculate the sensitivity, specificity, positive, and negative predictive values.

Methods and Material: The present study included 70 ascitic patients on top of Hepatitis $C$ virus over a period of 5 months from June 2016 to October 2016. Patients with hepatitis B virus, cirrhosis due to any other cause or receiving antibiotics two weeks before the study were excluded. The included patient were then divided into SBP group including 50 patients and non SBP group including 20 patients according to the gold standard to diagnose SBP which is PMNLs $\geq 250 \mathrm{cells} / \mathrm{mm}^{3}$. The Combi stik reagent strips dipped in $5 \mathrm{ml}$ of each sample of ascitic fluid and read visually to compare the color.

Results: In comparable with the gold standard for diagnosing SBP which is $P M N L \geq 250$ cells $/ \mathrm{mm}^{3}$ the using of Combi Stik leukocyte esterase reagent strips taking grade 3 as cutoff showed sensitivity, specificity, positive predictive value, negative predictive value and accuracy (100\%) with a very high statistically significance $(P=<0.001)$ with a highly positive correlation. A negative result of leukocyte esterase test excludes SBP with a high degree of certainty.

Conclusions: The diagnostic accuracy of leukocyte esterase reagent strips, comparable with the gold standard test, was excellent for excluding and diagnosing SBP in an emergency, it can be used as an easy and rapid bedside test for diagnosing SBP sitting.

Keywords: Ascites, liver cirrhosis, spontaneous bacterial peritonitis, polymorphnuclear leukocytes, leukocyte esterase reagent strips.
\end{abstract}




\section{INTRODUCTION}

Spontaneous bacterial peritonitis (SBP) is a frequent and severe complication of cirrhotic patients with ascites. The prevalence of SBP among unselected hospitalized cirrhotic patients with ascites ranges between $10 \%$ and $30 \%{ }^{(1)}$, Although antibiotic therapy produces a good response, the mortality of untreated SBP remains high (> 80\%), and a satisfactory patient course and clinical outcome is based on an aggressive approach aiming to rapid diagnosis and prompt initiation of antibiotic therapy ${ }^{(2)}$.The polymorphonuclear (PMN) leukocyte count in the ascitic fluid has proven to be a very useful and sensitive method for diagnosing SBP ${ }^{(3)}$. SBP is highly likely when the PMN cell count in the ascitic fluid reaches a cutoff of $250 / \mathrm{mm}^{3}$ and antibiotic therapy must be started immediately, without waiting for a culture from the ascitic fluid. ${ }^{(4)}$ However, total and PMN leukocyte counts from the ascitic fluid are not always available everywhere, or it may be impossible to obtain them in emergency situations. ${ }^{(3)}$ Many hospitals in developing countries have limited laboratory facilities, or are unable to perform PMN counts in ascitic fluid at night or over the weekend. Considering the mortality from SBP, this test will help to improve the management of SBP. It has been proposed that reagent strip testing for leukocyte esterase is rapid and could be utilized to reduce the time between performing paracentesis and obtaining a presumptive diagnosis of SBP from a few hours to a few seconds ${ }^{(5)}$. Moreover, such strips would be available everywhere, are easy to use, do not require expertise, thereby allowing antibiotic therapy to be immediately started ${ }^{(6)}$. Leukocyte esterase (LE) is an esterase (a type of enzyme) produced by leukocytes (white blood cells) (7) and is used as a urine test for the presence of white blood cell associated with infection. Reagent strip testing for leukocyte esterase had been found to be a sensitive and accurate predictor for the presence of PMN in body fluids such as urine, cerebrospinal fluid, seminal and peritoneal fluid ${ }^{(8)}$. This diagnostic method is useful for determining the effectiveness of the antibiotic therapy and could be a useful tool for diagnosing
SBP, especially in developing countries

(9)

Therefore, it seems very important to validate the use of such reagent strips in our country. The Leukocyte esterase test was based on the esterase activity of the leucocytes. A pyrrole, esterified with an amino-acid is used as the substrate; hydrolysis of the ester (mediated by the esterase) releases the pyrrole which in turn reacts with a diazonium salt present on the strip yielding a violet or purple azo dye in the relevant pad of the strip ${ }^{(10)}$. The main aim of this study is to emphasize the diagnostic accuracy of the Combi Stik leucocyte esterase reagent strips for rapid diagnosing SBP and The chief objective of the present study is to calculate the sensitivity, specificity, positive, and negative predictive values.

\section{SUBJECTS AND METHODS}

This study was an analytical cross sectional study included 70 ascitic patients with liver cirrhosis on top of Hepatitis C Virus (HCV) diagnosed by antibodies and PCR. The patients were collected randomly from the Internal Medicine departments at Cairo university hospital from June 2016 to October 2016. After following the ethical regulation of conducting the study on human participant, an informed consent was obtained from all patients. By considering PMNLs in ascitic fluid $\geq 250 / \mathrm{mm}^{3}$ as a gold standard for the diagnosis of SBP, patients divided into 20 patients diagnosed as non SBP based on PMNLs $<250$ cells $/ \mathrm{mm}^{3}$ and 50 patients were diagnosed SBP based on PMNLs $\geq 250$ cells / $\mathrm{mm}^{3}$. While exclusion criteria included Patients with ascites receiving antibiotics 2 weeks before paracentesis as it can alter the results, Ascitic patients with Hepatitis B Virus, Ascitic patients due to any other cause (malignancy, cardiac or tuberculosis) excluded by history and radiological findings. All patients were subjected to thorough history taking. Complete physical examination to detect stigmata of liver cirrhosis. Abdominal ultrasonography: For assessment of liver and spleen size, portal and splenic vein diameter and the degree of ascites. Laboratory investigations including: Complete blood picture carried by (Cell Dyn 3500; 
Spectra group). Urea, Creatinine, AST, ALT, Bilirubin, Serum albumin, Alkaline phosphatase(ALP), Gamma Glutamile transferase (GGT) and LDH were measured using (AU 480; Beckman Coulter) using its commercially available reagents. Prothrombin time, concentration and INR. Screening for virology markers: HCV antibodies were detected by ELIZA and HCV RNA by PCR. HBV surface antigen and HIV by ELIZA (to be excluded). Abdominal paracentesis was performed under strict sterile conditions with Sample of $30 \mathrm{~cm}$ of ascitic fluid is withdrawn and divided in to:-

1- Sample for PMN and total leukocyte count was collected in a heparin anticoagulant tube. Differential cell count and cytology were examined with a conventional optical microscope. A manual cell count with differential study was performed for all samples by experienced technicians.

2-Sample for appropriate biochemical tests (Glucose, Protein, Albumin, LDH) and SAAG was calculated in each sample by calculating the difference between serum albumin and ascitic albumin.

3- Cultures sample were seeded at bedside with the inoculation of $10 \mathrm{~mL}$ of ascitic fluid into aerobic and anaerobic media blood culture bottles (Bactec).

4- Sample for Combi Stik leukocyte esterase reagent strips (DFI-diagnostics) urine strips with a 4 grade colorimetric scale grading from grade 0 to 3 . This test pad contains an indoxyl ester and diazonium salt. It is followed by an azo-coupling reaction of the aromatic amine formed by leukocytes esterase with a diazonium salt on the reaction pad. The azo dye produced causes a color change from beige to violet. The reagent strip was immersed immediately in $5 \mathrm{~mL}$ of AF placed on a dry and clean container as described by the manufacturer for identification of leukocyte esterase. After $120 \mathrm{sec}$, the reagent strip was read comparing the color of the leukocyte reagent strip area with the colorimetric 4-grade scale depicted on the bottle. Based on the degree of color change in the reagent strip area; the results were scored as grade 0 or nil, grade 1 or traces, grade 2 or low, grade 3 or high as shown in figure 1 . We considered grade 0-1-2 which indicates $(0,+25,+70 \mathrm{WBC} / \mu \mathrm{l})$ as negative and grade $3(+++500 \mathrm{WBC} / \mu \mathrm{l})$ as positive and clinically significant. In all cases, physician was unaware of ascites manual cellular count. Results of leukocyte esterase reagent strip were compared with ascitic fluid PMN cell Count.

Statistical Methods: Data were coded and entered using the statistical package SPSS (Statistical Package for the Social Sciences) version 23. Sensitivity, specificity, positive predictive value of the test, negative predictive value of the test and accuracy were calculated. Chi-Square and Fischer Exact test have been used to find the significance of association of study parameters with HPE findings.

\section{RESULTS}

The 70 patients in this study included 41 males $(58.6 \%)$ and 29 females (41.4\%), the mean age of patients was $52.41 \pm 5.17$ years old ranging from 45 to 62 years old. Non SBP group included 20 patients $(28.6 \%)$ with mean age $52.65 \pm 5.4$ years old ranging from 45 to 62 years old, including 7 females $(35.0 \%)$ and 13 males $(65.0 \%)$. SBP group included 50 patients $(71.4 \%)$ with mean age $52.32 \pm 5.13$ years old ranging from 45 to 62 years old, including 22 females $(44.0 \%)$ and 28 males $(56.0 \%)$ as shown in table 1 .

Table 1: - Description of the studied population

\begin{tabular}{|lc|}
\hline Patients $(n)$ & 70 \\
Age (years), mean & $52.41 \pm 5.17$ \\
Male sex, $n(\%)$ & $41(58.6 \%)$ \\
SBP patients , n $(\%)$ & $50(71.4 \%)$ \\
Male SBP patients, $\mathrm{n}(\%)$ & $28(56 \%)$ \\
Non SBP patients, $\mathrm{n}(\%)$ & $20(28.6 \%)$ \\
Male non SBP patients, $\mathrm{n}(\%)$ & $13(65 \%)$ \\
\hline
\end{tabular}

\section{Age and sex distribution among studied group}

There was no statistically significant difference between two groups regarding age and $\operatorname{sex}(\mathrm{p}=0.59)$ Regarding clinical data; Non SBP group, by history all 20 patients had no recurrent hepatic encephalopathy (100\%), all 20 patients were off fever $(100 \%), 5$ patients with abdominal pain (25\%), 15 patients had no pain $(75 \%)$, only 1 patient had abdominal tenderness on examination (5\%) while 19 patients were free( $95 \%)$. 
According to SBP group, by history 24 patients had recurrent hepatic encephalopathy $(48.0 \%)$ and 26 patients had no recurrent hepatic encephalopathy (52\%), 47 patients had fever $(94.0 \%)$ and 3 patients had no fever $(6.0 \%)$, all 50 patients had abdominal pain and abdominal tenderness (100\%).

The comparison between the SBP group and non SBP group was statistically significant regarding the clinical presentation with $\mathrm{P}$ value $<0.001$ as shown in figure 2.

Fig 2: Graph for comparison of symptomatology between SBP and non SBP

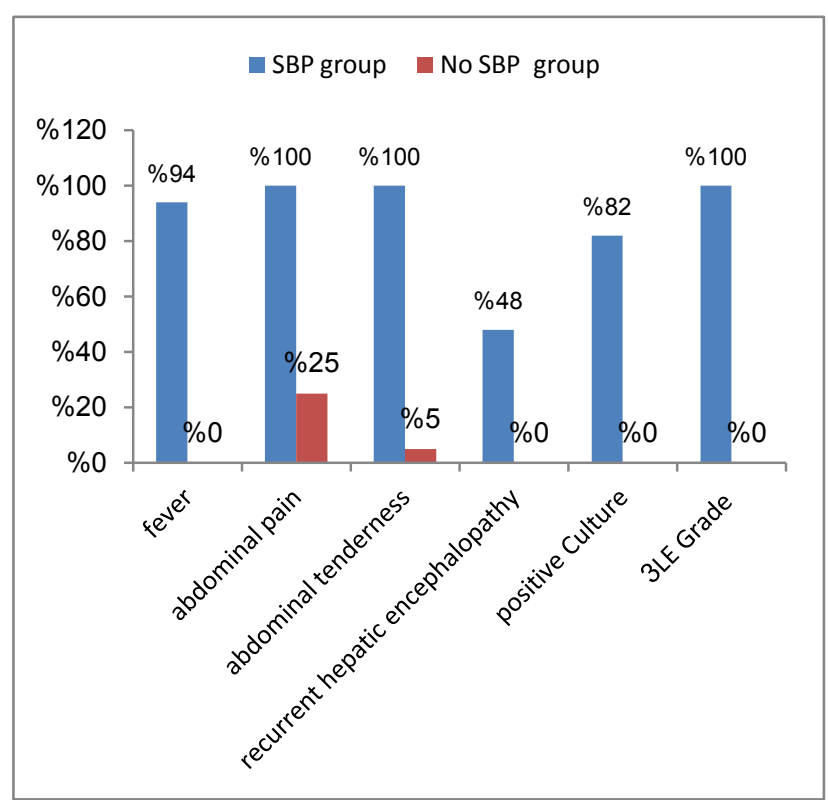

Regarding routine labs as shown in table 2, the comparison between the SBP group and non SBP group for serum Total leukocyte count (TLC), Platelets, Alkaline phosphatase, Gamma GT, Creatinine, Urea, Serum LDH, Total bilirubin, Direct bilirubin, Prothrombin time, Prothrombin concentration(PC), INR were statistically significant with $\mathrm{P}$ value $(<0.001,0.021,<0.001,0.002,0.004$, $0.002,<0.001,<0.001,0.002,0.006,0.010 \& 0.004$ respectively).However, the comparison between both groups for hemoglobin, ALT, AST, Serum albumin, Serum total protein were not statistically significant (table 2). The results of ascitic fluid analysis between SBP group and non SBP group was statistically significant regarding PMNLs, total protein (AF TP), albumin, $\mathrm{LDH}$ with $\mathrm{P}$ value $(<0.001,0.031,0.012$ and $<0.001$ respectively), While ascitic fluid glucose was not statistically significant. The Serum ascetic albumin gradient
(SAAG) in all non SBP group was $>1.1$, while in SBP group 3 patients were $<1.1$ and 47 patients were $>1$.1.the comparison between both groups was not statistically significant as shown in table 3 . The ascitic fluid culture results from all 70 patients, forty one patients were positive culture mainly for Escherichia coli and 29 patients were negative culture. All 41 patients with positive culture were SBP with PMNL $\geq 250$ cells $/ \mathrm{mm}$, while from the 29 patients with negative culture 20 patients were Non SBP with PMNLs $<250$ cell/ $\mathrm{mm}$ and 9 patients were SBP . By comparison between SBP group and Non SBP group According to ascitic fluid culture it was statistically significant with $(\mathrm{P}=<0.001)$ as shown in table 4. By using LE reagent strips, all patients in the non-SBP group had negative test. On the other hand the SBP group patients had strongly positive test with grade 3(Violet colour) as shown in figure 1. When using the LE strips as a diagnostic marker for SBP based on AF PMNLs, the sensitivity and specificity were $100 \%$ and $100 \%$ respectively. The positive predictivity, negative predictivity and accuracy were $100 \%, 100 \%$ and $100 \%$ respectively as shown in table 5 .

Fig 1: Colorimetric scale of LE reagent strips

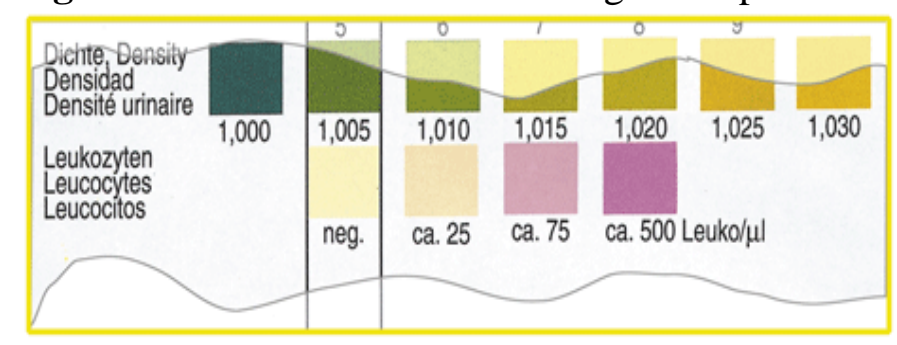


Table 2 : Comparison between routine labs in SBP and non SBP group

\begin{tabular}{|c|c|c|c|c|c|c|c|c|c|c|c|}
\hline & \multicolumn{5}{|c|}{ No SBP group $(\mathrm{n}=20)$} & \multicolumn{5}{|c|}{ SBP Group $(\mathrm{n}=50)$} & \multirow[t]{2}{*}{ P Value } \\
\hline & Mean & SD & Median & Minimum & Maximum & Mean & SD & Median & Minimum & Maximum & \\
\hline HB & 8.96 & 1.38 & 9.10 & 6.20 & 11.30 & 9.60 & 1.77 & 9.80 & 3.60 & 13.80 & .115 \\
\hline TLC & 6.08 & 1.95 & 6.33 & 2.3 & 8.9 & 16.48 & 6.61 & 16 & 1.6 & 32 & $<0.001$ \\
\hline PLT & 91.85 & 41.24 & 82.00 & 30.00 & 205.00 & 139.56 & 96.47 & 103.50 & 47.00 & 519.00 & .021 \\
\hline ALT & 32.90 & 19.24 & 24.50 & 13.00 & 73.00 & 42.56 & 26.29 & 34.00 & 10.00 & 107.00 & .093 \\
\hline AST & 61.30 & 20.01 & 62.50 & 20.00 & 93.00 & 84.10 & 92.42 & 57.50 & 12.00 & 552.00 & .745 \\
\hline ALB & 2.21 & .46 & 2.10 & 1.60 & 3.40 & 2.19 & .46 & 2.20 & 1.40 & 3.60 & 948 \\
\hline Alp & 108.35 & 63.54 & 100.00 & 34.00 & 337.00 & 211.60 & 90.62 & 200.00 & 41.00 & 477.00 & $<0.001$ \\
\hline GGT & 113.05 & 89.47 & 92.50 & 14.00 & 407.00 & 172.10 & 104.77 & 150.00 & 18.00 & 477.00 & .002 \\
\hline Creat & 1.53 & 1.33 & 1.05 & .50 & 5.80 & 2.39 & 1.54 & 1.90 & .50 & 6.57 & .004 \\
\hline Urea & 73.85 & 88.58 & 30.00 & 16.00 & 285.00 & 114.16 & 80.39 & 101.50 & 15.00 & 345.00 & .002 \\
\hline Tptn & 5.84 & .70 & 5.75 & 4.50 & 7.00 & 5.84 & .74 & 5.60 & 3.80 & 7.50 & 917 \\
\hline LDH & 123.55 & 31.24 & 120.00 & 80.00 & 200.00 & 347.58 & 130.52 & 350.00 & 130.00 & 940.00 & $<0.001$ \\
\hline T.bil & 2.57 & 2.31 & 1.80 & .80 & 10.00 & 6.11 & 5.45 & 4.30 & .43 & 30.40 & $<0.001$ \\
\hline D. bil & 1.27 & 1.18 & .80 & .20 & 5.00 & 3.23 & 3.90 & 1.70 & .09 & 22.13 & .002 \\
\hline PT & 16.63 & 3.11 & 17.00 & 12.00 & 22.50 & 19.67 & 3.63 & 18.45 & 14.00 & 27.00 & .006 \\
\hline PC & 58.48 & 19.12 & 50.85 & 35.00 & 95.00 & 44.81 & 11.28 & 45.00 & 20.00 & 67.00 & .010 \\
\hline INR & 1.59 & .50 & 1.48 & .90 & 2.60 & 2.25 & 1.58 & 1.95 & 1.14 & 12.00 & . 004 \\
\hline
\end{tabular}

Table 3: Comparison between ascitic fluid analysis in SBP and non SBP groups regarding ascitic fluid analysis

\begin{tabular}{|c|c|c|c|c|c|c|c|c|c|c|c|}
\hline & \multicolumn{5}{|c|}{ No SBP } & \multicolumn{5}{|c|}{ SBP } & \multirow{2}{*}{$\begin{array}{c}\mathrm{P} \\
\text { value }\end{array}$} \\
\hline & Mean & SD & Median & Minimum & Maximum & Mean & SD & Median & Minimum & Maximum & \\
\hline $\begin{array}{l}\mathrm{AF} \\
\text { PMNLs }\end{array}$ & 81.50 & 65.80 & 60.00 & 10.00 & 240.00 & 1866.82 & 1308.71 & 1630.00 & 320.00 & 5200.00 & $<0.001$ \\
\hline AF TP & 1.32 & .82 & 1.05 & .50 & 3.50 & 1.83 & 1.00 & 1.55 & .30 & 4.30 & .031 \\
\hline AF Alb & .37 & .27 & .30 & .10 & 1.00 & .62 & .47 & .50 & .10 & 1.97 & .012 \\
\hline $\begin{array}{l}\text { AF } \\
\text { glucose }\end{array}$ & 120.45 & 44.64 & 115.00 & 80.00 & 253.00 & 129.14 & 71.72 & 120.00 & 53.00 & 518.00 & .754 \\
\hline AF LDH & 53.30 & 17.85 & 50.50 & 30.00 & 89.00 & 267.58 & 182.40 & 230.00 & 39.00 & 920.00 & $<0.001$ \\
\hline SAAG & 1.84 & .51 & 1.75 & 1.10 & 2.90 & 1.59 & .48 & 1.50 & .54 & 2.70 & .096 \\
\hline
\end{tabular}

Table 4: Comparison between Ascitic fluid culture in SBP group and non SBP group

\begin{tabular}{|c|c|c|c|c|c|c|}
\hline & & \multicolumn{2}{|c|}{ No SBP group } & \multicolumn{2}{|c|}{ SBP group } & P value \\
\hline & & Count & $\%$ & Count & $\%$ & \\
\hline \multirow[t]{2}{*}{ Culture } & POSITIVE & 0 & $.0 \%$ & 41 & $82.0 \%$ & \multirow[t]{2}{*}{$<0.001$} \\
\hline & negative & 20 & $100.0 \%$ & 9 & $18.0 \%$ & \\
\hline
\end{tabular}

Table 5 : Sensitivity, Specificity, Positive predictivity, Negative predictivity and accuracy for Leukocyte esterase reagent strips in ascetic fluid in diagnosing SBP.

\begin{tabular}{|l|c|c|c|c|c|}
\hline Test Result Variable(s) & Sensitivity (\%) & Specificity (\%) & PPV (\%) & NPV (\%) & Accuracy (\%) \\
\hline LE Grade & 100 & 100 & 100 & 100 & 100 \\
\hline
\end{tabular}




\section{DISCUSSION}

Spontaneous bacterial peritonitis is one of the most severe complication in cirrhotic patients with ascites leading to increase hospitalization, renal failure and mortality, so early diagnosis and treatment is mandatory to decrease hospital stay and mortality. Paracentesis is recommended to all cirrhotic patient with ascites as many patients with SBP are asymptomatic. Currently, differential cell count of ascitic fluid is usually performed by a manual method using light microscopy and counting chambers. However, the diagnosis is often delayed when laboratory personnel are not readily available or in the private practice setting where specimens are sent to an offsite laboratory. This is a major drawback, as rapid diagnosis of SBP and immediate initiation of antibiotic treatment is of paramount importance to decrease mortality ${ }^{(11)}$. Leukocyte esterase enzyme has been shown as an important marker for PMN cell activity .In our study, we used Combi Stik reagent strips with a 4 grade colorimetric scale grading from grade 0 to 3 , in which we considered grade $0-1-2$ which indicates $(0$, $+25,+70 \mathrm{WBC} / \mu l)$ as negative and grade $3(+++500$ $\mathrm{WBC} / \mu \mathrm{l})$ as positive. All patients in the non SBP group had negative test. On the other hand, the SBP group patients had strongly positive test with grade 3. When using the LE strips as a diagnostic marker for SBP, the sensitivity and specificity was $100 \%$. The positive predictivity, negative predictivity and accuracy were $100 \%$. In patients with a reagent strip result of grade 0 , a diagnosis of ascitic fluid infection could confidently be ruled out because the NPV was $100 \%$. Oey and his colleagues had tested 153 ascitic samples from 53 patient using (Combur10 strips, Roche Diagnostics) with four different color shades corresponding to $0,25,100$ or 500 leukocytes/ $\mu 1$., one strip was read visually and one with a photospectrometer (Urisys 1100®, Roche Diagnostics), with visual reading, the sensitivity for diagnosing SBP was $75 \%$, the specificity $99 \%$, the positive predictive value $82 \%$ and the negative predictive value $98 \%$. The diagnostic accuracy for automated reading was slightly superior $(\mathrm{p}=0.007)$ : sensitivity $100 \%$, specificity $93 \%$, positive predictive value $55 \%$ and negative predictive value $100 \% .^{(12)}$ Our results by visual reading of Combi Stik strips were slightly similar to results of their study by automated method. Moorthy and his colleagues had tested 200 patients with ascites of unknown aetiology with the same mean age of our study, with exclusion of antibiotic intake in the last 48 hours, pregnant females ,previous surgery from the study, they used MultiStix 10 SG with 5 grade scale in their study, the results were The sensitivity and specificity of LER strip test in diagnosing SBP when grade 3 (>125 PMN's/cu ml) was taken as cut off point was $87.5 \%$ and $95.3 \%$ respectively, Positive predictive value and negative predictive values were $80 \%$ and $97.2 \%$ respectively. The sensitivity and specificity of LER strip test in diagnosing SBP when grade 4(>500 PMN's /cu ml.) was taken as cut off was $53.1 \%$ and $100 \%$, Positive predictive value and Negative predictive value were $100 \%$ and $90.8 \%{ }^{(9)}$, this means that when they used a higher grade, specificity and PPV increased but sensitivity and NPV were lower than using lower grade as cut-off, in spite of that when we used in our study another type of strips and took grade 3 (>500 leukocytes/cu $\mathrm{ml})$ as cutoff it gave equally higher sensitivity and specificity $100 \%$. Our results were largely consistent with Hashemian et al, who tested 100 patients with ascites due to cirrhosis by using (urine matrix 11 brand) with 5 grade scale and grade 3 considered as cutoff for diagnosis. Based on standard calculation, sensitivity, specificity, positive and negative predictive values, and accuracy based on the results were $95 \%, 96.30 \%, 85.0 \%, 97.5 \%$ and $95.0 \%$ respectively ${ }^{(13)}$. Kamal and his colleague investigated LE reagent strips as a rapid, easy and cheap test for the diagnosis of SBP, the study was done on 100 patients with cirrhosis and ascites with same inclusion and exclusion criteria of our study, they used (Multistix 10SGR, Bayer Diagnostics) with 5 grades. The sensitivity, specificity, positive predictive value, negative predictive value of leukocyte esterase dipstick test to diagnose SBP were $89.8 \%, 92.7 \%, 94.6 \%, 82 \%$ respectively ${ }^{(14)}$. Our results were superior to this study for utilization 
of LE strips in rapid diagnosis of SBP this may be attributed to the type of reagent strip we used with lower grade scale (4 grades). Bafandeh has evaluated the diagnostic value of LE reagent strips on 132 patient with cirrhosis and ascites using (Medi-Test combi, France) and the results showed that sensitivity, specificity, PPV and NPV of leukocyte esterase reagent strip test were $97.5 \%$, $84.6 \%, 74 \%$ \& $98.7 \%$ respectively $^{(5)}$. Our results were slightly similar according to sensitivity and NPV and superior according to specificity and PPV. These inconsistent results could be related to variability in reagent strips, patient populations, different cut-off values and the subjective interpretation of the reagent strip result. However, the consistent high negative predictive value could make the reagent strips a very useful rule-out tool. The results of these studies were comparable with our results in terms of a high negative predictive value of reagent strip testing. A negative test result, strongly predicts absence of SBP. Thus, in patients undergoing diagnostic paracentesis, a negative reagent strip result may imply that further diagnostic studies - polymorphonuclear neutrophil count and bacterial cultures - are not useful and can be omitted.

\section{CONCLUSIONS}

Early diagnosis of SBP is mandatory to decrease morbidity and mortality in cirrhotic patient with ascites. Leukocyte esterase reagent strip test is easy, does not require expertise, and is rapid, can be performed bedside with a low cost, and has high sensitivity and specificity for exclusion or diagnosis of SBP. In addition, saving time at diagnosis of SBP leads to rapid start of antibiotic therapy.

\section{FINANCIAL SUPPORT AND SPONSERSHIP} Nil.

\section{CONFLICTS OF INTEREST}

There are no conflicts of interest

\section{REFERENCES}

1. Runyon BA. AASLD. Introduction to the revised American Association for the Study of Liver Diseases Practice Guideline management of adult patients with ascites due to cirrhosis. Hepatol 2013;57: 1651-3.

2. Koulaouzidis A. Diagnosis of spontaneous bacterial peritonitis:An update on leucocyte esterase reagent strips. World J Gastroenterol 2011; 17(9): 1091-4.

3. Garcia Tsao G. spontaneous bacterial peritonitis: historical perspective. J Hepatol 2004; 41:522-7.

4. Wong C, Holroyd-Leduc J, Thorpe K et al., Does this patient have bacterial peritonitis or portal hypertension? How do perform a paracentesis and analyze the results? JAMA 2008; 299:1166-78.

5. Bafandeh Y, Khodaei M. Evaluation of leukocyte esterase reagent strip test to detect spontaneous bacterial peritonitis in cirrhotic patients. Gastroenterology Insights. PAGE Press Publications; 2012 Jun 6;4(1):13.

6. Sithara K. Balagopal , Ashik Sainu ., Varghese Thomas. Evaluation of leucocyte esterase reagent strip test for the rapid bedside diagnosis of spontaneous bacterial peritonitis Indian J Gastroenterol 2010 (March-April):29(2):74-77

7. Braga LL, Souza MH, Barbosa AM et al., Diagnosis of spontaneous bacterial peritonitis in cirrhotic patients in northeastern Brazil by use of rapid urinescreening test. Sao Paulo Med J. 2006 May 4; 124(3):141-4.

8. Nguyen-Khac E, Cadranel JF, Thevenot T. Review article: the utility of reagent strips in the diagnosis of infected ascites in cirrhotic patients. Aliment Pharmacol Ther. 2008;28: 282-8.

9. Moorthy S., Chalasani V., Ramakrishnan S.R. et al., Efficiency Assessment of Leucocyte Esterase Reagent strips in rapid bedside diagnosis of Spontaneous Bacterial Peritonitis - a comparison study with the 
gold standard Absolute Neutrophil Counts in ascitic fluid. Indian journal of applied research 2015;5: 465-7.

10. Sapey T, Mena E, Fort E, et al., Rapid diagnosis of spontaneous bacterial peritonitis with leukocyte esterase reagent strip in a European and American center Journal of gastroenterolhepatol 2005;20;187-92

11. Fernandes S.R., Santos P., Fatela N. et al., Ascitic Calprotectin is a Novel and Accurate Marker for Spontaneous Bacterial Peritonitis. J. Clin. Lab. Anal. 2016; 30(6):1139-1145.

12. Oey R.C. , Kuiper J.J., Van Buuren H.R. et al., Reagent strips are efficient to rule out spontaneous bacterial peritonitis in cirrhotics. Netherlands J.of medicine 2016;74(6):257261.

13. Hashemian A.M., Koorosh A., Moghaddam H.Z. et al., Diagnostic Value of Leukocyte Esterase Test Strip Reagents for Rapid Clinical Diagnosis of Spontaneous Bacterial Peritonitis in Patients Admitted to Hospital Emergency Departments in Iran.Iran Red Crescent Med J.2015;17(10): e 21341.

14. Kamal E. and Abdelhaleem H. Leukocyte esterase reagent (LER) strips: A rapid, easy and cheap test for the diagnosis of spontaneous bacterial peritonitis. MJMR, 2013;24(1):1-6. 